\title{
Method Comparison For Estimation Of Distributed Parameters In Permittivity Models Using Reflectance
}

\author{
H.T. Banks, Jared Catenacci and Shuhua Hu \\ Center for Research in Scientific Computation \\ North Carolina State University \\ Raleigh, NC 27695-8212 USA
}

May 22, 2015

\begin{abstract}
The use of reflectance spectroscopy is a current area of study for the non-invasive evaluation of complex materials such as ceramic matrix composites. In order to model the reflectance, one must specify a model for the complex permittivity. In this work we compare two methods for modeling the complex permittivity of a heterogenous material. In one approach, we impose a probability distribution on a subset of the dielectric parameters. This approach leads to an infinite dimensional optimization problem over the space of probability measures. We approximate this space with a finite dimensional space by using either a Dirac approximation method or a linear spline approximation method. The second approach is to assume a number of oscillators in the permittivity model, and then use a convolution with a normal distribution. We compare both of these approaches on simulated data sets as well as data obtained from inorganic glasses. Each of these methods are able to fit the data well, yet the ease in interpreting the estimation results of imposing a probability distribution on parameters, as well as the tight mathematical results $[2,7]$ guaranteeing convergence under the Prohorov metric, lead us to favor the first approach.
\end{abstract}

Key Words: inverse problems, nonlinear regression, mathematical model approximation.

Mathematics Subject Classification: 34A55, 62G07, 62.G08 


\section{Introduction}

There is a current interest in the integration of ceramic matrix composites (CMCs) for both static and rotating components in high temperature turbine engines, specifically in highperformance aircraft engines and other gas turbine engines [1,19]. Over the course of a CMCs lifetime, oxidation occurs which can compromise the integrity of the desired material properties. Collaborators at Wright-Patterson Air Force Base have hypothesized that as the CMC under study (a ceramic matrix with a silicon carbide fiber) is exposed to high temperatures, components of the material will transition from an amorphous to crystalline state. Thus, there is a need for noninvasive techniques which can quantify the degradation, and possibly also the level of oxidation. Fourier Transform Infrared (FTIR) spectroscopy has been investigated as one possible non destructive evaluation tool with the potential to quantify the oxidation behavior $[10,16,18,20]$. Due to the fact that CMCs are optically dense, we will consider the reflectance (rather than transmission or absorption) spectroscopy.

Our goal is to develop a technique for modeling the reflectance, obtained using an FTIR spectrometer, which can be used to quantify the levels of degradation. In modeling reflectance, it is customary to assume a specific combination of polarization models with a predetermined number of dielectric parameters. However, due to the highly heterogenous nature of CMCs, the number of dielectric mechanisms are unknown. In a case where the material under study is inorganic glass, a convolution of the Lorentz and Gaussian functions (a linear combination of normal distributions is imposed on the resonance frequency in the Lorentz model) was proposed by Efimov, et al., as early as in 1985 (e.g., see [12,13]) (we will refer to this as the Efimov approach). Another possible approach to deal with this difficulty, which was investigated in $[4,5]$, is to impose an unknown probability distribution on the dielectric parameters. In that work, a distribution was imposed on the resonance wavenumber and we continue that convention in our current investigation. There is a solid theoretical foundation for the non-parametric estimation of a probability distribution $[2,3,6,7,17]$ under the Prohorov Metric Framework (PMF). The estimation procedure involves approximating the space of admissible probability measures by a finite dimensional space using, for example, either a Dirac approximation method or a linear spline approximation method.

In this work we compare the two available approximation schemes under the PMF, Dirac masses and piecewise linear splines, to establish the accuracy and reliability for the estimation of the distribution of resonance wavenumbers. Additionally, we also carryout the Efimov approach of imposing a normal distribution of the resonance wavenumbers and compare these results with those obtained using the PMF. In Section 2, we give the permittivity models which are embedded in the model for the reflection coefficient, and establish the foundation for the two approximation schemes under the PMF. In Section 3 we give the results obtained using both synthetic data sets and experimental data sets obtained from various inorganic glasses. Finally, in Section 5 we conclude the paper with summary remarks and plans for future work. 


\section{The model for the complex permittivity and the re- flection coefficient}

The Lorentz model is derived by considering the polarization which results from the displacement of electrons from equilibrium under the effect of an applied electromagnetic field. The Lorentz model for the complex relative permittivity with a single-resonance is given by

$$
\widehat{\varepsilon}_{r}(\omega)=\varepsilon_{\infty}-\frac{\omega_{p}^{2}}{\omega^{2}-i \omega / \tau_{f}-\omega_{0}^{2}} .
$$

In the above equation, $\varepsilon_{\infty}$ denotes the relative permittivity of the medium at infinite frequency, $\tau_{f}$ is the relaxation time, and $\omega_{p}=\omega_{0} \sqrt{\varepsilon_{s}-\varepsilon_{\infty}}$ is called the plasma frequency of the medium, where $\omega_{0}$ is the resonance frequency, and $\varepsilon_{s}$ is the relative permittivity of the medium at zero frequency, also known as the "static" dielectric constant.

In practice it is typical for the data to be collected as a function of $k$, the wavenumber, rather than frequency $\omega$. Using the relationship that $k=\omega /(2 \pi c)$, where $c$ is the speed of light, we obtain the relative permittivity as a function of wavenumber

$$
\widehat{\varepsilon}_{r}(k)=\varepsilon_{\infty}-\frac{k_{p}^{2}}{k^{2}-i k / \tau_{k}-k_{0}^{2}} .
$$

In the above equation $k_{p}=k_{0} \sqrt{\varepsilon_{s}-\varepsilon_{\infty}}, k_{0}=\omega_{0} /(2 \pi c)$, and $\tau_{k}=2 \pi c \tau_{f}$. We will refer to $k_{0}$ as the resonance wavenumber and we will omit the subscript on the relaxation time $\tau_{k}$ when it is clear that we are referring to the relaxation time for the permittivity in terms of wavenumber.

According to quantum mechanical dispersion theory, and allowing for a material to contain multiple oscillators, the more general model for the permittivity can be given by

$$
\widehat{\varepsilon}_{r}(k)=\varepsilon_{\infty}-\sum_{j=1}^{J} \frac{S_{j}}{k^{2}-i k / \tau_{j}-k_{0_{j}}^{2}},
$$

where $S_{j}$ is understood to be the intensity of the $j$ th oscillator. The intensities $S_{j}$ are sometimes replaced by the contributions of the oscillators, $\Delta \varepsilon_{0_{j}} k_{0_{j}}^{2}=S_{j}$, where

$$
\sum_{j=1}^{J} \Delta \varepsilon_{0_{j}}=\varepsilon_{s}-\varepsilon_{\infty}
$$

\subsection{Efimov model for permittivity}

In $[11,14]$, Efimov describes an observed band broadening in the spectra of glasses, which he contributes to the random distribution of particular realizations of microscopic structures. To handle this band broadening, Efimov chooses to approximate the broadening by using a Gaussian probability density function. This leads to a model for the relative permittivity given by

$$
\widehat{\varepsilon}_{r}(k)=\varepsilon_{\infty}-\sum_{j=1}^{J} \frac{S_{j}}{\sqrt{2 \pi} \sigma_{j}} \int_{-\infty}^{\infty} \frac{\exp \left(-\left(x-k_{0_{j}}\right)^{2} / 2 \sigma_{j}^{2}\right)}{k^{2}-i k / \tau_{j}-x^{2}} d x
$$


where $J$ is the number of oscillators. We note that Efimov has made the tacit assumption to consider the intensities $S_{j}$ as a "free" parameter. By this we mean, that had the relationship $S_{j}=\Delta \varepsilon_{0_{j}} k_{0_{j}}^{2}$ been enforced, then there would be a $x^{2}$ term multiplying the exponential function in the integration.

Efimov notes that the band broadening could be better approximated by a truncated Gaussian in order to ensure that the wavenumber ranges remain non-negative. We make this modification which results in what we will refer to as the modified Efimov relative permittivity model, given by

$$
\widehat{\varepsilon}_{r}(k ; \theta)=\varepsilon_{\infty}-\sum_{j=1}^{J} \frac{S_{j}}{c_{j}} \int_{0}^{\infty} \frac{\exp \left(-\left(x-k_{0_{j}}\right)^{2} / 2 \sigma_{j}^{2}\right)}{k^{2}-i k / \tau_{j}-x^{2}} d x,
$$

where

$$
c_{j}=\int_{0}^{\infty} \exp \left(-\left(x-k_{0_{j}}\right)^{2} / 2 \sigma_{j}^{2}\right) d x
$$

In the above equation $\theta=\left(\varepsilon_{\infty},\left\{S_{j}, \tau_{j}, k_{0_{j}}, \sigma_{j}\right\}_{j=1}^{J}\right)^{T} \in \Theta \subset \mathbb{R}^{4 J+1}$ with $\Theta$ assumed to be compact.

\subsection{Prohorov metric framework model for permittivity}

An alternate method which can be used to account for multiple dielectric mechanisms present in a material, is to impose a probability distribution on the dielectric parameters, or a subset of the dielectric parameters. Here, we take this approach and then will make use of the PMF to non-parametrically estimate the distribution(s). In this work we only consider the case where a distribution is placed on the resonance wavenumbers (distributions could also be put on the relaxation constants $\tau$-see [4] .

To allow for a distribution $G$ of resonance wavenumbers over an admissible set $\mathcal{K} \subset \mathbb{R}$, we generalize the relative permittivity for the Lorentz model (2.2) to be

$$
\widehat{\varepsilon}_{r}(k ; G, \theta)=\varepsilon_{\infty}-\int_{\mathcal{K}} \frac{k_{p}^{2}}{k^{2}-i k / \tau-k_{0}^{2}} d G\left(k_{0}\right),
$$

where $G \in \mathcal{P}(\mathcal{K})$, the set of admissible probability measures on $\mathcal{K}$. In the case of assuming a distribution of resonance wavenumbers we also have the constant parameter vector $\theta=$ $\left(\varepsilon_{s}, \varepsilon_{\infty}, \tau\right)^{T} \in \Theta$ with $\Theta \subset \mathbb{R}^{3}$ assumed to be compact.

We remark the the Efimov model is not a subcase of the PMF models.

\subsection{Reflection coefficient}

We now turn our attention to obtaining a model for the reflectivity. For simplicity, we assume that a monochromatic uniform wave of wavenumber $k$ is incident on a plane interface between free space and a dielectric medium. We will deal with data which is obtained either at an incident angle of $\phi=45^{\circ}$ or $0^{\circ}$. Both situations can be accurately described by assuming that the reflectance is composed of the parallel and perpendicular polarizations in equal weights. Thus we obtain the equation for the reflectivity

$$
R(k ; G, \theta)=\frac{1}{2}\left(\left|r_{\perp}(k ; G, \theta)\right|^{2}+\left|r_{\|}(k ; G, \theta)\right|^{2}\right),
$$


where

$$
r_{\perp}(k ; G, \theta)=\frac{\cos \phi-\sqrt{\widehat{\varepsilon}_{r}(k ; G, \theta)-\sin \phi}}{\cos \phi+\sqrt{\widehat{\varepsilon}_{r}(k ; G, \theta)-\sin \phi}},
$$

and

$$
r_{\|}(k ; G, \theta)=\frac{\sqrt{1-\sin ^{2} \phi / \widehat{\varepsilon}_{r}(k ; G, \theta)}-\sqrt{\widehat{\varepsilon}_{r}(k ; G, \theta)} \cos \phi}{\sqrt{1-\sin ^{2} \phi / \widehat{\varepsilon}_{r}(k ; G, \theta)}+\sqrt{\widehat{\varepsilon}_{r}(k ; G, \theta)} \cos \phi} .
$$

Notice that if $\phi=0^{\circ}$, then the equation for the reflectivity reduces to $R(k ; G, \theta)=\left|r_{\perp}(k ; G, \theta)\right|^{2}$. A full derivation of the reflection coefficient can be found in many electromagnetic treatments (e.g., see [9, Section 9.3]).

At this point we remark that when using the modified Efimov model for the complex permittivity, the distribution $G$ is absent. In order to avoid cumbersome notation, when the modified Efimov model is used, we will ignore the input $G$ mathematically, but not drop it notationally.

\subsection{Statistical model}

Our goal is to estimate both the unknown probability measure $G$ as well as the additional model parameters when using the PMF approach. Of course, when using the Efimov model we need only estimate the relevant model parameters. We consider a statistical model of the form

$$
Y_{j}=R\left(k_{j} ; G_{0}, \theta_{0}\right)+\mathcal{V}_{j}, \quad j=0,1,2, \ldots, n .
$$

In the above equation $Y_{j}$ is a random variable which is composed of the reflectance with $G_{0}$ the "true" probability measure and $\theta_{0}$ the "true" parameters at a sampling wavenumber $k_{j}$, and the measurement error $\mathcal{V}_{j}$. For simplicity, we consider that the errors $\mathcal{V}_{j}$ are independent and identically distributed with mean 0 and constant variance.

\subsection{Inverse problem}

With the assumptions we have made for the measurement errors in the statistical model, the estimates $\widehat{G}$ of $G$ and $\widehat{\theta}$ of $\theta$ can be obtained through an ordinary least squares formulation

$$
(\widehat{G}, \widehat{\theta})=\underset{(G, \theta) \in(\mathcal{P}(\mathcal{K}) \times \Theta)}{\arg \min } J(G, \theta) .
$$

In the above equation, the cost functional $J$ is defined as

$$
J(G, \theta)=\sum_{j=0}^{n}\left(R\left(k_{j} ; G, \theta\right)-y_{j}\right)^{2}
$$

and $y_{j}$ is a realization of $Y_{j}, j=0,1, \ldots, n$ in $(2.12)$. That is,

$$
y_{j}=R\left(k ; G_{0}, \theta_{0}\right)+\nu_{j}, \quad j=0,1,2, \ldots, n .
$$

We note that (2.13) is an infinite-dimensional optimization problem in the case of using the PMF. Thus, we need to approximate the infinite dimensional space $\mathcal{P}(\mathcal{K})$ with a finite 
dimensional space $\mathcal{P}^{N}(\mathcal{K})$ in order to have a computationally tractable finite-dimensional optimization problem

$$
(\widehat{G}, \widehat{\theta})=\underset{(G, \theta) \in\left(\mathcal{P}^{N}(\mathcal{K}) \times \Theta\right)}{\arg \min } J(G, \theta) .
$$

We will consider two finite-dimensional spaces, $\mathcal{P}_{D}^{N}(\mathcal{K})$ and $\mathcal{P}_{S}^{N}(\mathcal{K})$, to approximate $\mathcal{P}(\mathcal{K})$. The space $\mathcal{P}_{D}^{N}$ involves the use of Dirac measures, and the space $\mathcal{P}_{S}^{N}$ involves the use of piecewise linear splines. We define these two spaces as

$$
\mathcal{P}_{D}^{N}(\mathcal{K})=\left\{G \in \mathcal{P}(\mathcal{K}) \mid G=\sum_{m=1}^{N} \alpha_{m} \Delta_{x_{m}}, \text { where } \alpha_{m} \geq 0 \text { and } \sum_{m=1}^{N} \alpha_{m}=1\right\}
$$

and

$$
\mathcal{P}_{S}^{N}(\mathcal{K})=\left\{G \in \mathcal{P}(\mathcal{K}) \mid G^{\prime}=\sum_{m=1}^{N} \alpha_{m} l_{m}\left(k_{0}\right), \text { where } \alpha_{m} \geq 0 \text { and } \sum_{m=1}^{N} \alpha_{m} \int_{\mathcal{K}_{m}} l_{m}(\xi) d \xi=1\right\}
$$

where $\Delta_{x_{m}}$ is a Dirac measure with atom at $x_{m}$, and $l_{m}$ is the $m$ th linear spline element with support $\mathcal{K}_{m}$. With either of these spaces we have reduced the infinite-dimensional problem to a finite-dimensional problem in which we only need to estimate $\theta$ and the weights $\boldsymbol{\alpha}=$ $\left\{\alpha_{m}\right\}_{m=1}^{N}$. Following the work in [4] we will estimate the Dirac atom locations $\mathbf{x}=\left\{x_{m}\right\}_{m=1}^{N}$ as well. Hence, when using the Delta approximation method we have the minimization problem

$$
(\widehat{\boldsymbol{\alpha}}, \widehat{\mathbf{x}}, \widehat{\theta})=\underset{(\boldsymbol{\alpha}, \mathbf{x}, \theta) \in\left(\mathbb{R}_{D}^{N} \times \mathcal{K}^{N} \times \Theta\right)}{\arg \min } J\left(\sum_{m=1}^{N} \alpha_{m} \Delta_{x_{m}}, \theta\right)
$$

where

$$
\begin{aligned}
& \mathbb{R}_{D}^{N}=\left\{\boldsymbol{\alpha}=\left(\alpha_{1}, \alpha_{2}, \ldots, \alpha_{N}\right)^{T} \mid \alpha_{m} \geq 0, \text { and } \sum_{m=1}^{N} \alpha_{m}=1\right\}, \\
& \mathcal{K}^{N}=\left\{\mathbf{x}=\left(x_{1}, x_{2}, \ldots, x_{N}\right)^{T} \mid x_{m} \in \mathcal{K}, m=1,2, \ldots, N\right\} .
\end{aligned}
$$

Using the spline method we have the minimization problem

$$
(\widehat{\boldsymbol{\alpha}}, \widehat{\theta})=\underset{(\boldsymbol{\alpha}, \theta) \in\left(\mathbb{R}_{S}^{N} \times \Theta\right)}{\arg \min } J(G, \theta), \quad G^{\prime}=\sum_{m=1}^{N} \alpha_{m} l_{m}\left(k_{0}\right)
$$

where

$$
\mathbb{R}_{S}^{N}=\left\{\boldsymbol{\alpha}=\left(\alpha_{1}, \alpha_{2}, \ldots, \alpha_{N}\right)^{T} \mid \alpha_{m} \geq 0, \text { and } \sum_{m=1}^{N} \alpha_{m} \int_{\mathcal{K}_{m}} l_{m}(\xi) d \xi=1\right\}
$$

When using the modified Efimov model, we simply have the standard minimization problem

$$
\widehat{\theta}=\underset{\theta \in \Theta}{\arg \min } J(\theta)
$$

where $\Theta \subset \mathbb{R}^{4 J+1}$ is compact. 


\section{Results}

The results section is laid out as follows. First we investigate the differences between the Dirac and spline approximation methods and the modified Efimov approach using simulated data sets. We consider two simulated data sets, the first using a "true" distribution $G_{0}$ which is discrete, and the second distribution being continuous. Next we compare the methods using reflectance data obtained from three different inorganic glasses.

For the modified Efimov model we must choose the number of oscillators $J$ which describe the interrogated material. This is done by starting with a low value of oscillators and then increasing $J$ until the model fit gives a reasonable approximation to the data.

\subsection{Simulated data}

The simulated data was generated by evaluating (2.15) at $k=600+j \Delta k$, where $\Delta k=0.8$ and $j=0,1,2, \ldots, n=1000$. The errors, $\nu_{j}$, were chosen as a realization of a normally distributed random variable with mean 0 and standard deviation $\sigma_{0}=0.001$. The number of interrogating wavenumbers which we use here is similar to sampling capabilities of a modern FTIR spectrometer.

As noted above, for the true distribution $G_{0}$, we consider two cases. In the first case we take $G_{0}$ to be a discrete distribution, which is depicted as the true distribution (along with a number of graphs for the results from optimized PMF based fits-to-data) in Figure 2 below. In the second case we take $G_{0}$ to be a continuous distribution. For this we chose to take $G_{0}$ as a truncated bivariate normal distribution which can be seen in Figure 4 (again along with a number optimized fits-to-data). For both cases we used the scalar parameters $\theta_{0}=\left(\varepsilon_{s_{0}}, \varepsilon_{s_{0}}, \tau_{0}\right)=(1.6,1.32,0.017)$ and the incident angle was set to $\phi=45^{\circ}$.

\subsubsection{Discrete distribution}

The "true" discrete distribution $G_{0}$ used to simulate the data has 30 Dirac measures. There are two regions, between 650 and $1100 \mathrm{~cm}^{-1}$, and between 1100 and $1400 \mathrm{~cm}^{-1}$, in which the jump discontinuities present in the distribution produce relatively small increases. At $k_{0}=1100 \mathrm{~cm}^{-1}$ there is a relatively large jump of 0.34 . It is reasonable assumption to expect a distribution of similar characteristics to describe a CMC which is in a crystalline state.

In Figure 1 we give the model fit for both PMF approximation schemes using $N=45$ as well as for the modified Efimov approach with $J=6$. We see that both PMF methods obtain an excellent model fit, and similar fits were obtained for other values of $N$. The model using the modified Efimov approach fits the data well except for wavenumbers $k>1350 \mathrm{~cm}^{-1}$. We present the estimated distributions using the spline and Dirac methods in Figure 2 and the estimated scalar parameters can be found in Table 1.

We see an interesting feature as $N$ is increased using the Dirac approximation. With a low number of nodes, specifically for $N=15$ and 25, the estimation results match the true parameters and distribution very well. However, for $N$ greater than 25 , the estimation results begin to deviate from the true values. This is most likely due to an over parameterization of the problem. This increased freedom allows for the approximate model to begin to fit the noise present in the data. 


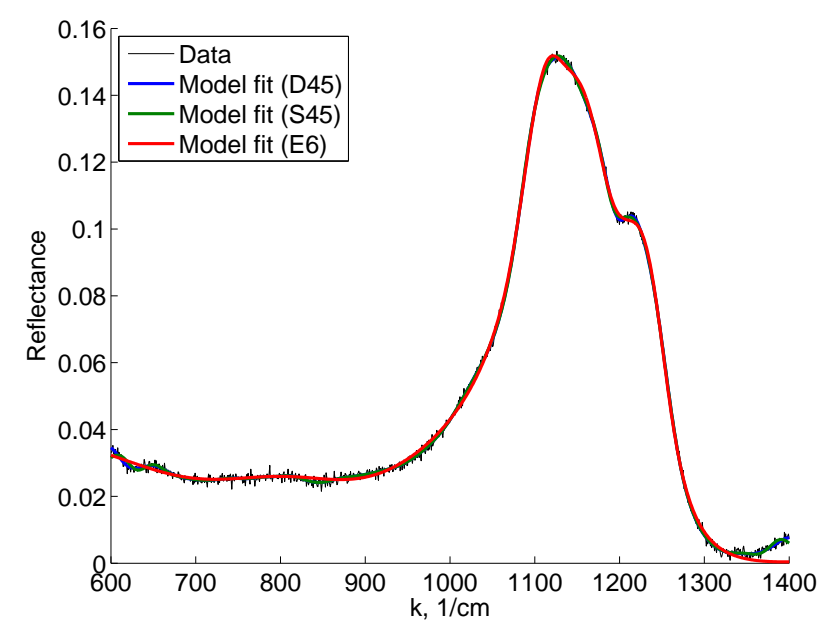

Figure 1: The model fits to the simulated data generated with a discrete distribution. The model fit using the Dirac approximation scheme is labeled as D45 and the spline approximation schemes as S45, where 45 is the number of nodes $N$, and the model fit using the modified Efimov method is labeled as E6 where $J=6$ oscillators were used.
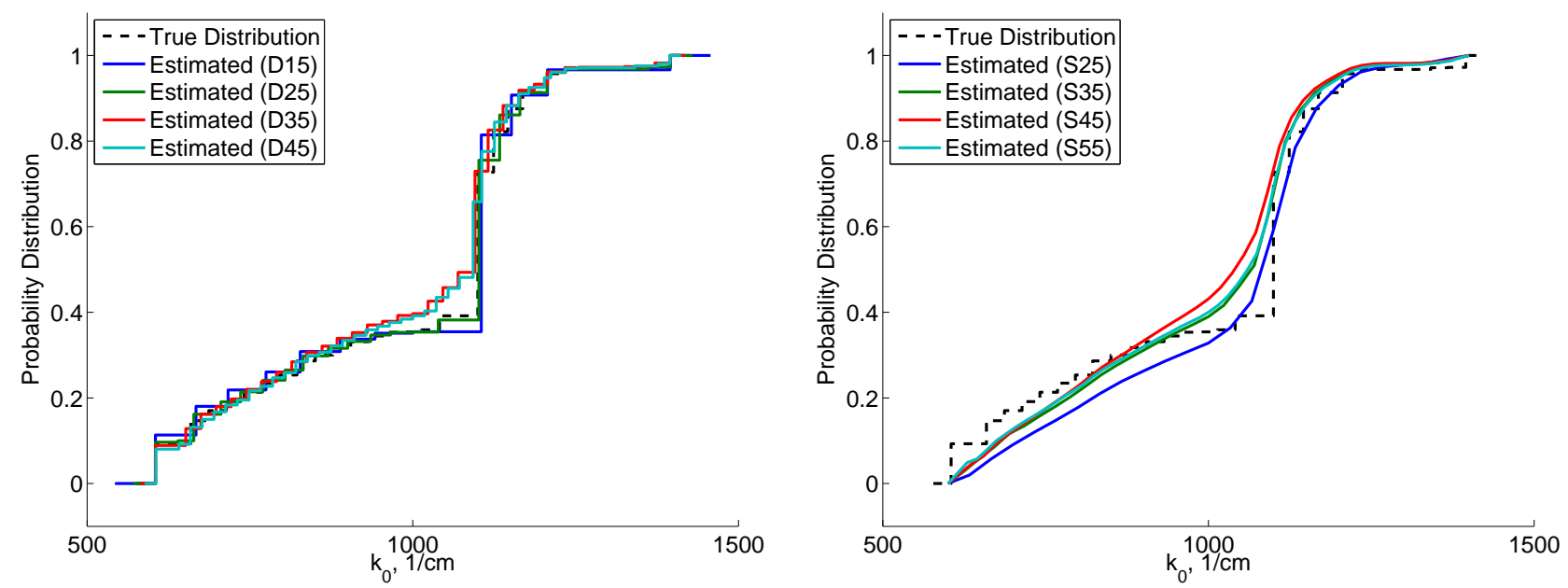

Figure 2: The estimated distributions to the simulated data using a discrete distribution using the Dirac approximation method with $N=15,25,35$ and 45 nodes (left) and using the spline approximation method with $N=25,35,45$ and 55 nodes (right).

The estimated distribution using the spline method is not able to replicate the large jump at $k_{0}=1100 \mathrm{~cm}^{-1}$, even when using as many as $N=55$ nodes. However, in general, the estimated distributions are reasonable approximations to the true distribution. This result is somewhat unexpected since we only can guarantee convergence of the spline method if the probability density function is absolutely continuous [8]. The estimated scalar values are all over estimated, and in particular the estimated values of $\tau$ are not close to the true value. This is consistent with the results in [4], in which it was shown that it is extremely difficult to accurately estimate the relaxation time $\tau$. 


\begin{tabular}{|c|ccc|c|ccc|c|cc|}
\hline \multicolumn{4}{|c|}{ Delta Approximation } & \multicolumn{5}{c|}{ Spline Approximation } & \multicolumn{3}{|c|}{ Efimov Method } \\
\hline$N$ & $\varepsilon_{s}$ & $\varepsilon_{\infty}$ & $\tau$ & $N$ & $\varepsilon_{s}$ & $\varepsilon_{\infty}$ & $\tau$ & $N$ & $\varepsilon_{s}$ & $\varepsilon_{\infty}$ \\
\hline 15 & 1.6187 & 1.3299 & 0.0142 & & & & & 8 & 1.9850 & 1.6000 \\
25 & 1.6055 & 1.3252 & 0.0164 & 25 & 1.7243 & 1.4931 & 0.0513 & & & \\
35 & 1.5707 & 1.2869 & 0.0192 & 35 & 1.7043 & 1.4496 & 0.0280 & & & \\
45 & 1.5668 & 1.2860 & 0.0194 & 45 & 1.6786 & 1.4228 & 0.0353 & & & \\
& & & & 55 & 1.7068 & 1.4502 & 0.0332 & & & \\
\hline$\theta_{0}$ & 1.6 & 1.32 & 0.017 & $\theta_{0}$ & 1.6 & 1.32 & 0.017 & $\theta_{0}$ & 1.6 & 1.32 \\
\hline
\end{tabular}

Table 1: The estimated parameters using the Dirac and spline approximation methods for the discrete distribution.

\begin{tabular}{|c|cccc|}
\hline Oscillator $(j)$ & $S_{j}$ & $\tau_{j}$ & $k_{0_{j}}$ & $\sigma_{j}$ \\
\hline 1 & $6.2021 \mathrm{e}+04$ & 0.0060 & 600.22 & 88.37 \\
2 & $1.9846 \mathrm{e}+04$ & 0.0135 & 839.29 & 56.89 \\
3 & $1.6175 \mathrm{e}+02$ & 0.0064 & 839.87 & 119.82 \\
4 & $2.7413 \mathrm{e}+04$ & 0.0968 & 1103.13 & 14.17 \\
5 & $1.7871 \mathrm{e}+05$ & 0.0124 & 1123.55 & 19.92 \\
6 & $1.3596 \mathrm{e}+04$ & 0.0317 & 1207.65 & 17.02 \\
\hline
\end{tabular}

Table 2: The estimated values of the intensities $S_{j}$, the relaxation times $\tau_{j}$, the resonance wavenumbers $k_{0_{j}}$ and the standard deviations $\sigma_{j}$ for each oscillator using the modified Efimov approach, using the simulated data with a discrete distribution.

In Table 2 we give the estimated values for the individual oscillators using the modified Efimov model. We expect to see an oscillator centered near $1100 \mathrm{~cm}^{-1}$ with a narrow broadening (i.e. a small standard deviation) to describe the jump discontinuity in the distribution. Indeed, we see that the $4 t h$ oscillator is centered at $k_{0_{4}}=1103.13 \mathrm{~cm}^{-1}$ and has a standard deviation of $\sigma_{j}=14.17$. Unexpectedly we also see that the 5 th and $6 t h$ oscillators also have a narrow broadening. Furthermore, using the Efimov approach, it is difficult to associate the oscillator directly to the size of the jump discontinuity in the distribution, one must look at the magnitude of the intensity $S_{j}$ relative to the other intensities to understand the relative "importance" of each oscillator. That is, from Table 2 we would deduce that the $5 t h$ oscillator with an intensity on the order to $10^{5}$ has more importance compared to the $3 r d$ oscillator which has an intensity of $10^{2}$ three orders of magnitude lower.

\subsubsection{Continuous distribution}

In this example we consider the case where the true distribution $G_{0}$ is taken as a truncated bivariate normal distribution with corresponding probability density function $g_{0}$ given by

$$
G_{0}\left(k_{0}\right)=\frac{\beta}{\sigma_{1} \sqrt{2 \pi}} \exp \left(-\frac{\left(k_{0}-\mu_{1}\right)^{2}}{2 \sigma_{1}^{2}}\right)+\frac{\beta}{\sigma_{2} \sqrt{2 \pi}} \exp \left(-\frac{\left(k_{0}-\mu_{2}\right)^{2}}{2 \sigma_{2}^{2}}\right), \quad k_{0} \in\left[\underline{k}_{0}, \bar{k}_{0}\right] .
$$


In the above equation, we take $\mu_{1}=850 \mathrm{~cm}^{-1}, \mu_{2}=1050 \mathrm{~cm}^{-1}, \sigma_{1}=70, \sigma_{2}=60, \underline{k}_{0}=600$ $\mathrm{cm}^{-1}, \bar{k}_{0}=1400 \mathrm{~cm}^{-1}$ and $\beta$ is the normalizing constant

$$
\beta^{-1}=\int_{\underline{k}_{0}}^{\bar{k}_{0}} \frac{1}{\sigma_{1} \sqrt{2 \pi}} \exp \left(-\frac{\left(k_{0}-\mu_{1}\right)^{2}}{2 \sigma_{1}^{2}}\right)+\frac{1}{\sigma_{2} \sqrt{2 \pi}} \exp \left(-\frac{\left(k_{0}-\mu_{2}\right)^{2}}{2 \sigma_{2}^{2}}\right) d k_{0} .
$$

We expect that a CMC in an amorphous state would best be represented by a continuous distribution.

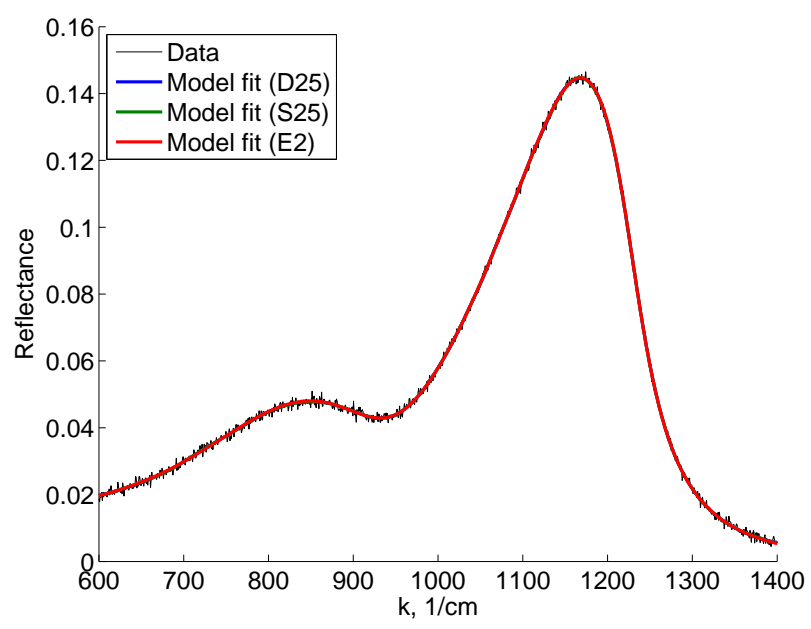

Figure 3: The model fits to the simulated data generated with a continuous distribution. For the Dirac and spline approximation schemes the number of nodes was taken as $N=25$ (labeled as D25 and S25 respectively) and for the Efimov approach we have $J=2$ (labeled as E2).

\begin{tabular}{|c|ccc|c|ccc|c|cc|}
\hline \multicolumn{4}{|c|}{ Delta Approximation } & \multicolumn{4}{c|}{ Spline Approximation } & \multicolumn{3}{|c|}{ Efimov Method } \\
\hline$N$ & $\varepsilon_{s}$ & $\varepsilon_{\infty}$ & $\tau$ & $N$ & $\varepsilon_{s}$ & $\varepsilon_{\infty}$ & $\tau$ & $N$ & $\varepsilon_{s}$ & $\varepsilon_{\infty}$ \\
\hline 10 & 1.4519 & 1.1613 & 0.0101 & 10 & 1.9299 & 1.7017 & 0.0264 & 2 & 1.5747 & 1.3013 \\
15 & 1.4107 & 1.1239 & 0.0112 & 15 & 1.5768 & 1.3040 & 0.0156 & & & \\
20 & 1.4011 & 1.1174 & 0.0116 & 20 & 1.5751 & 1.3074 & 0.0195 & & & \\
25 & 1.3909 & 1.1090 & 0.0124 & 25 & 1.5717 & 1.3077 & 0.0261 & & & \\
\hline$\theta_{0}$ & 1.6 & 1.32 & 0.017 & $\theta_{0}$ & 1.6 & 1.32 & 0.017 & $\theta_{0}$ & 1.6 & 1.32 \\
\hline
\end{tabular}

Table 3: The estimated parameters using the Dirac and spline approximation methods for the simulated data with a continuous distribution.

In Figure 3, we see that both PMF methods achieve a very good fit to the data where we set $N=25$. Using the Dirac method, we see in Figure 4 that the estimated distribution using $N=10$ nodes is shifted to the right of the true distribution. As $N$ is increased, the estimated distribution becomes a reasonable approximation of the continuous distribution. The estimated distribution using the spline method gives an excellent approximation of the true distribution except when $N=10$. In this case, there simply are not enough elements 

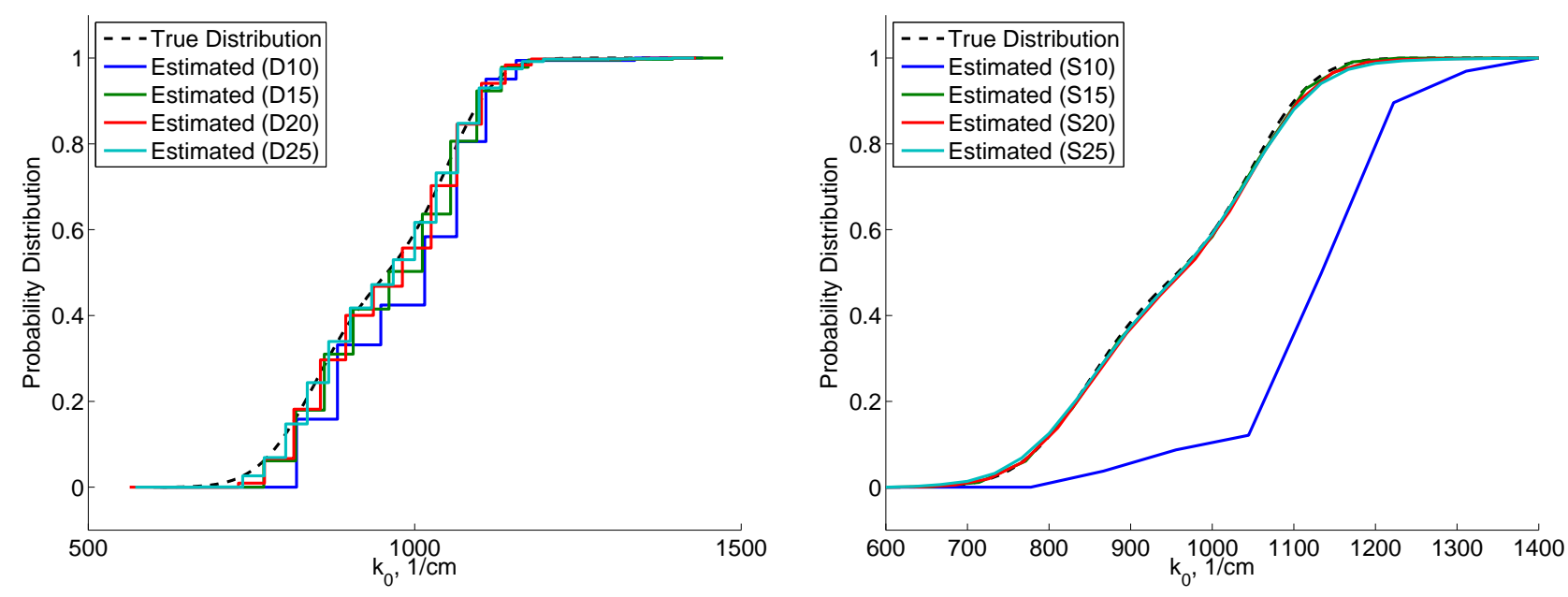

Figure 4: The estimated distributions to the simulated data using a continuous distribution using the Dirac approximation method (left) and using the spline approximation method (right). For both methods we chose the number of nodes to be $N=10,15,20$ and 25 .

\begin{tabular}{|c|cccc|}
\hline Oscillator $(j)$ & $S_{j}\left(1 \times 10^{5}\right)$ & $\tau_{j}$ & $k_{0_{j}}$ & $\sigma_{j}$ \\
\hline 1 & 1.0170 & 0.0139 & 861.23 & 66.61 \\
2 & 1.4794 & 0.0175 & 1057.06 & 60.07 \\
\hline
\end{tabular}

Table 4: The estimated values of the intensities $S_{j}$, the relaxation times $\tau_{j}$, the resonance wavenumbers $k_{0_{j}}$ and the standard deviations $\sigma_{j}$ for each oscillator using the modified Efimov approach using the simulated data with a continuous distribution.

in the approximation scheme to accurately fit the data. The Efimov approach is able to accurately fit the data with only $J=2$ oscillators, which is not surprising since the true distribution is composed of two normal distributions.

From Table 3, we see that the constant parameters are more accurately estimated using the spline approximation scheme and the Efimov approach. However, again the value for $\tau$ is difficult to estimate correctly.

In Table 4 we see that we obtain a good estimate for the relaxation time for the second oscillator, but not for the first. The resonance wavenumbers and the standard deviations for both oscillators are a good estimation of the mean and standard deviations of the bivariate normal distribution.

\subsection{Inorganic glass data}

To compare our approximation methods on experimental data sets, we present results obtained using inorganic glass data available in [11]. These inorganic glasses have properties similar to the materials which comprise the matrix in many CMCs. For these data sets the incident angle is approximately $\phi \approx 0^{\circ}$. 


\subsubsection{Vitreous Silica}

We first consider reflectivity data collected from Vitreous Silica in the 200 to $1350 \mathrm{~cm}^{-1}$ range (see Table A2 in [11]). In Figure 5 we present the model fits to the data using the Dirac and spline approximations with $N=50$ for both methods and using the modified Efimov method with $J=8$. We see that all of the methods are able to obtain a good fit to the data.

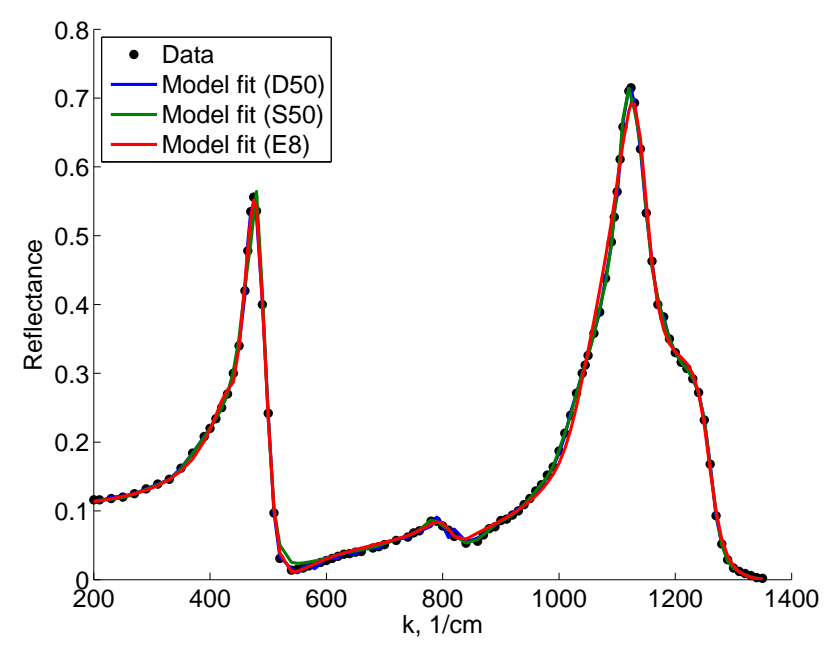

Figure 5: The model fits to the Vitreous Silica data.. For the Dirac and spline approximation schemes the number of nodes was taken as $N=50$ (labeled as D50 and S50 respectively) and for the Efimov approach we have $J=8$ (labeled as E8).
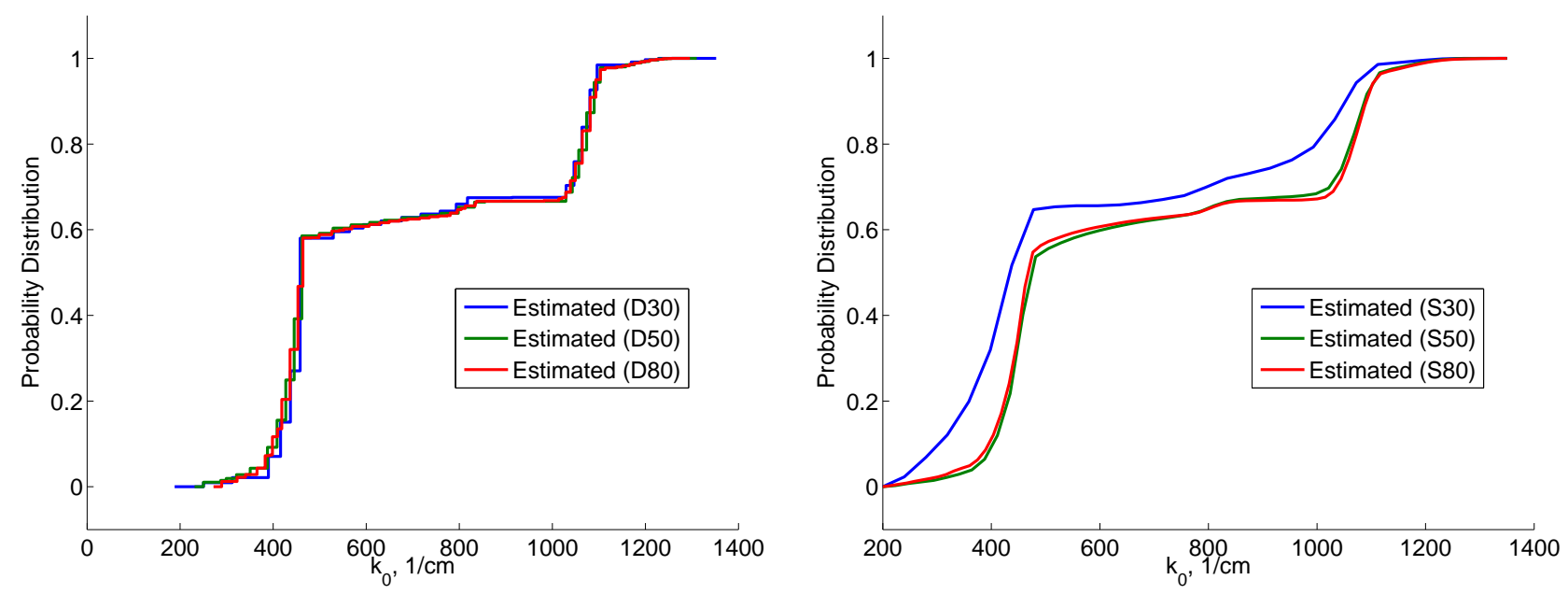

Figure 6: The estimated distributions to the Vitreous Silica data using the Dirac approximation method (left) and using the spline approximation method (right). For both methods we chose the number of nodes to be $N=30,50$ and 80 .

In Figure 6 we present the estimated distributions using both PMF methods. It is clear the the Dirac method gives consistent results for $N=30,50$ and 80, indicating that the 
method has converged for a relatively low value of $N$. The spline method gives consistent results for $N=50$ and 80; the distribution obtained using $N=30$ has the same general characteristic shape as the other two distributions, but it is clearly an outlier. Thus, we may assume that the spline method has not converged at $N=30$, but has by $N=50$. In fact, once both methods have converged, they have converged to the same distribution. Although the estimated distribution has large jumps near $k_{0}=400 \mathrm{~cm}^{-1}$ and $1050 \mathrm{~cm}^{-1}$, we agin see the surprising result that the spline approximation method is able to handle these regions of rapid change in the distribution.

\begin{tabular}{|c|ccc|c|ccc|c|cc|}
\hline \multicolumn{4}{|c|}{ Delta Approximation } & \multicolumn{4}{c|}{ Spline Approximation } & \multicolumn{3}{|c|}{ Efimov Method } \\
\hline$N$ & $\varepsilon_{s}$ & $\varepsilon_{\infty}$ & $\tau$ & $N$ & $\varepsilon_{s}$ & $\varepsilon_{\infty}$ & $\tau$ & $N$ & $\varepsilon_{s}$ & $\varepsilon_{\infty}$ \\
\hline 30 & 3.7535 & 2.0845 & 0.0398 & 30 & 3.3489 & 1.5553 & 0.1032 & 8 & 3.7530 & 2.1384 \\
50 & 3.7183 & 2.0487 & 0.0522 & 50 & 3.7079 & 2.0581 & 0.2020 & & & \\
80 & 3.6780 & 2.0153 & 0.0564 & 80 & 3.7019 & 2.0675 & 0.1851 & & & \\
\hline$\theta_{0}$ & 3.8 & 2.1 & & $\theta_{0}$ & 3.8 & 2.1 & & $\theta_{0}$ & 3.8 & 2.1 \\
\hline
\end{tabular}

Table 5: The estimated parameter values using the Dirac and spline approximation methods to fit the Vitreous Silica data. The "true" parameter values $\theta_{0}$ are the experimental values taken from [15].

\begin{tabular}{|c|cccc|}
\hline Oscillator $(j)$ & $S_{j}\left(1 \times 10^{5}\right)$ & $\tau_{j}$ & $k_{0_{j}}$ & $\sigma_{j}$ \\
\hline 1 & $3.6176 \mathrm{e}+03$ & 0.0183 & 311.63 & 66.62 \\
2 & $1.4231 \mathrm{e}+05$ & 0.0190 & 435.59 & 4.89 \\
3 & $4.4941 \mathrm{e}+04$ & 0.8445 & 459.17 & 8.71 \\
4 & $1.8884 \mathrm{e}+04$ & 0.0165 & 677.56 & 87.54 \\
5 & $2.7526 \mathrm{e}+04$ & 0.0282 & 806.88 & 9.48 \\
6 & $1.4019 \mathrm{e}+03$ & 0.0178 & 1014.97 & 34.98 \\
7 & $5.2605 \mathrm{e}+05$ & 1.6468 & 1077.90 & 21.29 \\
8 & $1.1559 \mathrm{e}+05$ & 0.0373 & 1166.09 & 46.83 \\
\hline
\end{tabular}

Table 6: The estimated values of the intensities $S_{j}$, the relaxation times $\tau_{j}$, the resonance wavenumbers $k_{0_{j}}$ and the standard deviations $\sigma_{j}$ for each oscillator using the modified Efimov approach on the Vitreous Silica data.

In Table 5, we present the estimated parameter values using both PMF methods. Notice that the estimated values of $\varepsilon_{s}$ and $\varepsilon_{\infty}$ are very similar for $N=30,50$ and 80 using the Dirac approximation method. However, the values for $\varepsilon_{s}$ and $\varepsilon_{\infty}$ using the spline method do not match the values obtained using the Dirac approximation at $N=30$, but do for $N=50$ and 80. Thus, again indicating that the Dirac method converges for a lower value of $N$ than the spline method. We also see that the high and low frequency limits are well approximated by the modified Efimov method.

The estimated values of the individual oscillators using the modified Efimov method are given in Table 6 . The $2 n d, 3 r d$ and 5 th oscillators, centered at approximately 435, 459 and $806 \mathrm{~cm}^{-1}$, respectively, each have a very narrow broadening. The 2 nd and $3 \mathrm{rd}$ oscillators correspond to the first large jump in the estimated distribution using the PMF methods. 
The 5 th oscillator is present in a region where the estimated distribution does not contain a sharp jump.

\subsubsection{Vitreous Germania}

We next consider reflectivity data collected from Vitreous Germania in the 200 to 1350 $\mathrm{cm}^{-1}$ range (see Table A7 in [11]). In Figure 7 we present the model fit and the estimated distributions using both the Dirac and spline methods with $N=50$ and using the modified Efimov method with $J=8$. Once again, we obtain very good fits to the data in all cases.

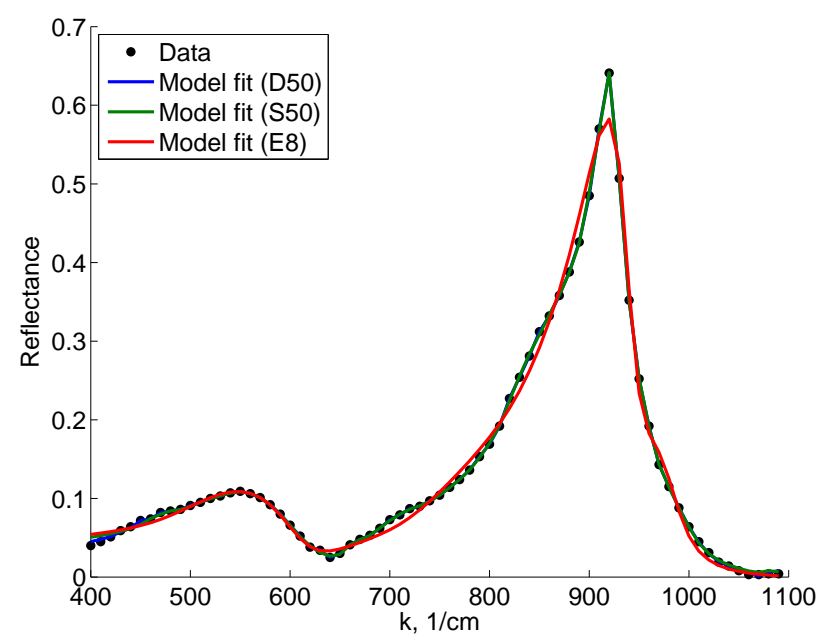

Figure 7: The model fits to the Vitreous Germania data. For the Dirac and spline approximation schemes the number of nodes was taken as $N=50$ (labeled as D50 and S50 respectively) and for the Efimov approach we have $J=8$ (labeled as E8).
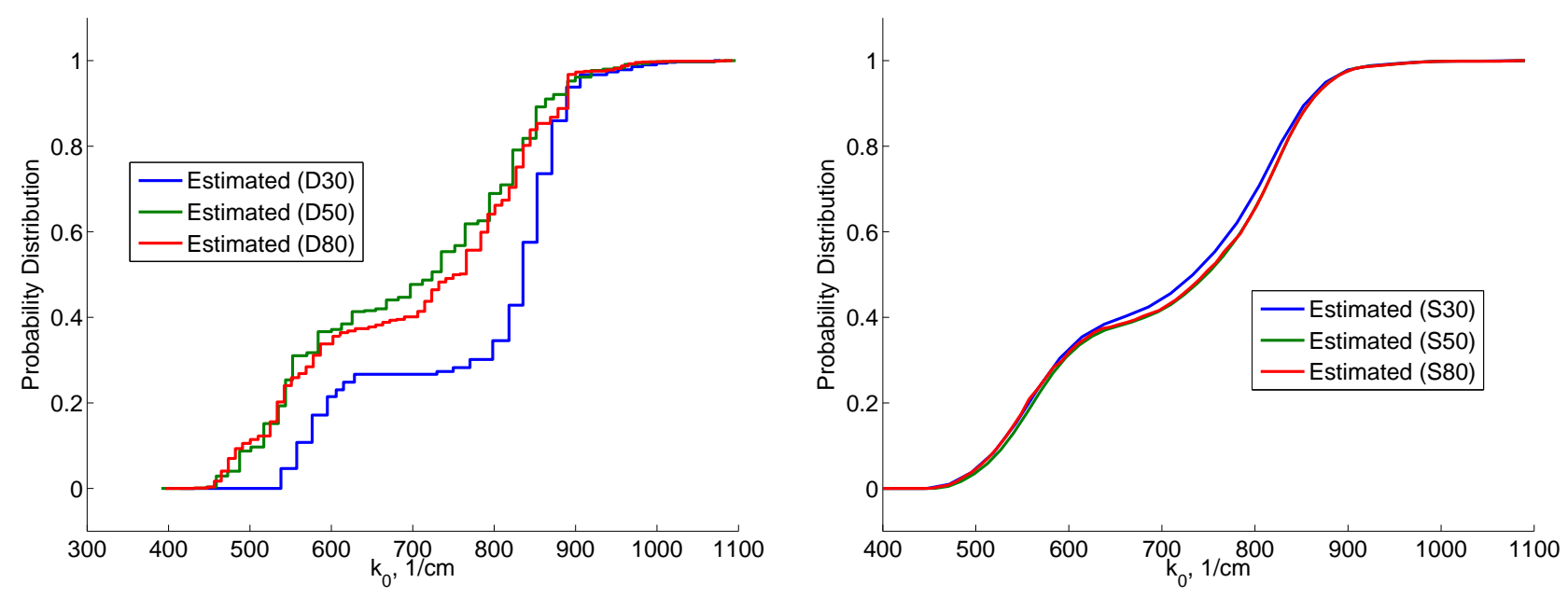

Figure 8: The estimated distributions to the Vitreous Germania data using the Dirac approximation method (left) and using the spline approximation method (right). For both methods we chose the number of nodes to be $N=30,50$ and 80 . 
From Figure 8 we see that each estimation using the spline method gives consistent results, and the estimation with $N=30$ using the Dirac method does not match the results using $N=50$ and 80 . In this case, the results suggest that the spline method converges for a lower number of nodes than the Dirac method, but both methods do converge to the same distribution. This should be expected since it appears as if the estimated distribution is sufficiently smooth, and it is known [8] that the spline method will outperform the Dirac method in this case.

In Table 7 we present the estimated parameter values for both methods. This time we see that the spline method gives consistent values for $\varepsilon_{s}$ and $\varepsilon_{\infty}$, whereas for $N=30$, the values estimated using the Dirac method are the outliers. The estimated values for $\varepsilon_{s}$ and $\varepsilon_{\infty}$ using the modified Efimov approach are slightly higher than the estimates using the PMF methods.

\begin{tabular}{|c|ccc|c|ccc|c|cc|}
\hline \multicolumn{4}{|c|}{ Delta Approximation } & \multicolumn{4}{c|}{ Spline Approximation } & \multicolumn{3}{c|}{ Efimov Method } \\
\hline$N$ & $\varepsilon_{s}$ & $\varepsilon_{\infty}$ & $\tau$ & $N$ & $\varepsilon_{s}$ & $\varepsilon_{\infty}$ & $\tau$ & $N$ & $\varepsilon_{s}$ & $\varepsilon_{\infty}$ \\
\hline 30 & 2.3992 & 1.7707 & 0.0779 & 30 & 2.0034 & 1.3320 & 0.4989 & 8 & 2.1844 & 1.5200 \\
50 & 1.8961 & 1.3013 & 0.7741 & 50 & 2.0567 & 1.3854 & 0.2581 & & & \\
80 & 1.9068 & 1.3435 & 0.6250 & 80 & 2.0254 & 1.3659 & 0.4964 & & & \\
\hline
\end{tabular}

Table 7: The estimated parameter values using the Dirac and spline approximation methods to fit the Vitreous Gernamia data.

\begin{tabular}{|c|cccc|}
\hline Oscillator $(j)$ & $S_{j}\left(1 \times 10^{5}\right)$ & $\tau_{j}$ & $k_{0_{j}}$ & $\sigma_{j}$ \\
\hline 1 & $1.5434 \mathrm{e}+03$ & 0.0159 & 511.79 & 68.73 \\
2 & $2.6232 \mathrm{e}+03$ & 0.0178 & 540.71 & 51.28 \\
3 & $5.9389 \mathrm{e}+04$ & 0.1661 & 565.51 & 35.91 \\
4 & $4.4435 \mathrm{e}+04$ & 0.0160 & 696.72 & 68.21 \\
5 & $1.4581 \mathrm{e}+05$ & 0.6777 & 812.01 & 40.25 \\
6 & $9.9376 \mathrm{e}+04$ & 1.1347 & 852.97 & 30.93 \\
7 & $6.1715 \mathrm{e}+02$ & 0.0157 & 940.72 & 69.75 \\
8 & $7.1409 \mathrm{e}+03$ & 0.4162 & 958.32 & 19.93 \\
\hline
\end{tabular}

Table 8: The estimated values of the intensities $S_{j}$, the relaxation times $\tau_{j}$, the resonance wavenumbers $k_{0_{j}}$ and the standard deviations $\sigma_{j}$ for each oscillator using the modified Efimov approach on the data obtain from Vitreous Germania.

In Table 8 we present the individual oscillator estimates obtain from the modified Efimov approach. In this case we see that the only oscillator which has a somewhat narrow broadening is present at $958 \mathrm{~cm}^{-1}$. This oscillator has an intensity of $10^{3}$, two orders of magnitude lower than the largest estimated intensity. This indicates that the modified Efimov approach agrees with the results seen using the PMF, that there are no sharp jumps in the resonance wavenumber which characterize the Vitreous Germania data. 


\subsubsection{Sodium Silicate}

As a final consideration, we use reflectivity data collected from Sodium Silicate in the 40 to $1260 \mathrm{~cm}^{-1}$ range (see Table A3 in [11]). In Figure 9 we give the model fit to the data and the estimated distributions using the Dirac and spline approximation schemes using $N=25$ and using the modified Efimov approach with $J=9$.

We see that the estimated distributions using both methods agree very well for the relatively low number of nodes, and the agreement is increased for $N=30$ as is seen from Figure 10. Additionally, in Table 9 we see that the estimated values of $\varepsilon_{s}$ and $\varepsilon_{\infty}$ agree well for $N=30$. Thus, it appears in this case that both the spline and Dirac approximation methods have converged at a relatively low number of nodes. It should be noted, that for this particular data set, there were 62 data points, which is why we did not use a larger number of nodes than $N=30$. Using the modified Efimov approach, the estimated value of $\varepsilon_{\infty}$ is consistent with the results using the PMF, but the value of $\varepsilon_{s}$ does not match.

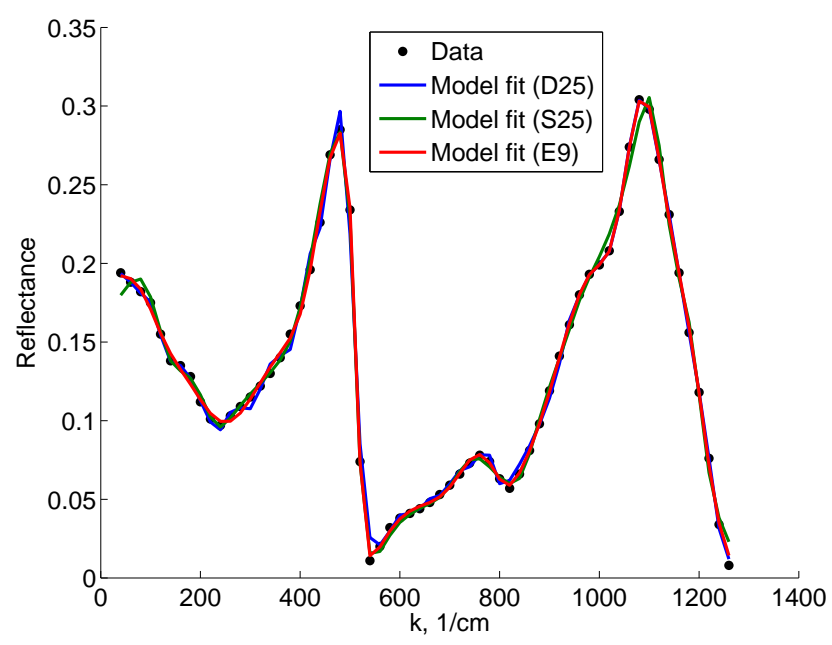

Figure 9: The model fits to the Sodium Silicate data. For the Dirac and spline approximation schemes the number of nodes was taken as $N=25$ (labeled as D25 and S25 respectively) and for the Efimov approach we have $J=9$ (labeled as E9).

\begin{tabular}{|c|ccc|c|ccc|c|cc|}
\hline \multicolumn{4}{|c|}{ Delta Approximation } & \multicolumn{4}{c|}{ Spline Approximation } & \multicolumn{3}{c|}{ Efimov Method } \\
\hline$N$ & $\varepsilon_{s}$ & $\varepsilon_{\infty}$ & $\tau$ & $N$ & $\varepsilon_{s}$ & $\varepsilon_{\infty}$ & $\tau$ & $N$ & $\varepsilon_{s}$ & $\varepsilon_{\infty}$ \\
\hline 25 & 6.3266 & 2.1110 & 0.0200 & 25 & 5.7623 & 1.5496 & 0.0269 & 9 & 7.4048 & 2.1179 \\
30 & 5.9747 & 2.0939 & 0.0240 & 30 & 5.9068 & 1.9573 & 0.0305 & & & \\
\hline
\end{tabular}

Table 9: The estimated parameter values using the Dirac and spline approximation methods as compared to the Efimov method to fit the Sodium Silicate Silica data.

In Table 10 we present the estimation results for the oscillators using the modified Efimov approach. In this case, the oscillator with the most narrow broadening is the 8th oscillator which is centered at $1057 \mathrm{~cm}^{-1}$. This oscillator corresponds to a region of relatively gradual increase in the estimated distribution. 

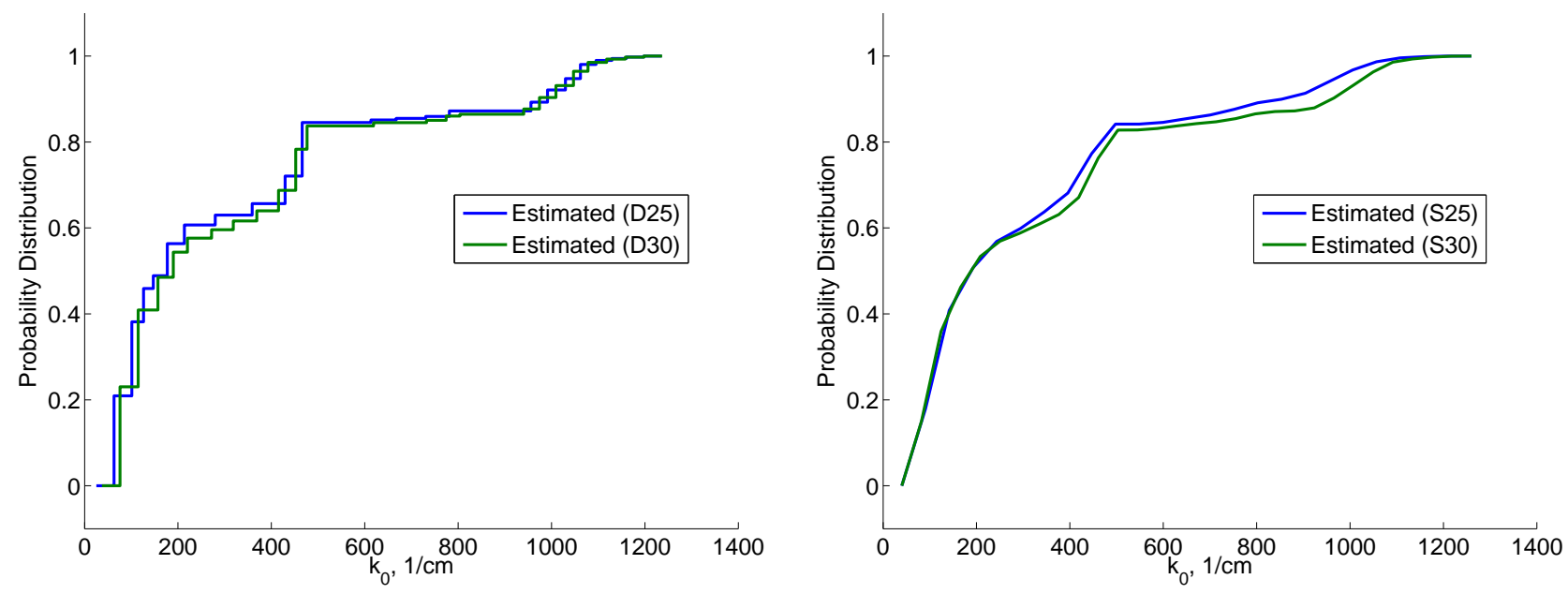

Figure 10: The estimated distributions to the Sodium Silicate data using the Dirac approximation method (left) and using the spline approximation method (right). For both methods we chose the number of nodes to be $N=25$ and 30 .

\begin{tabular}{|c|cccc|}
\hline Oscillator $(j)$ & $S_{j}\left(1 \times 10^{5}\right)$ & $\tau_{j}$ & $k_{0_{j}}$ & $\sigma_{j}$ \\
\hline 1 & $8.7256 \mathrm{e}+03$ & 0.0191 & 95.82 & 24.06 \\
2 & $3.7072 \mathrm{e}+04$ & 0.0208 & 187.11 & 62.71 \\
3 & $7.1957 \mathrm{e}+04$ & 0.0292 & 412.24 & 53.15 \\
4 & $9.0464 \mathrm{e}+04$ & 0.0764 & 459.40 & 23.43 \\
5 & $2.6822 \mathrm{e}+04$ & 0.0279 & 638.16 & 53.20 \\
6 & $3.9439 \mathrm{e}+04$ & 0.0491 & 778.93 & 29.66 \\
7 & $1.8828 \mathrm{e}+05$ & 0.5529 & 986.26 & 40.27 \\
8 & $2.7424 \mathrm{e}+05$ & 0.0154 & 1057.79 & 13.43 \\
9 & $9.9229 \mathrm{e}+04$ & 0.0775 & 1122.55 & 65.13 \\
\hline
\end{tabular}

Table 10: The estimated values of the intensities $S_{j}$, the relaxation times $\tau_{j}$, the resonance wavenumbers $k_{0_{j}}$ and the standard deviations $\sigma_{j}$ for each oscillator using the modified Efimov approach on the Sodium Silicate data. 


\section{Concluding remarks and future work}

In this work we consider two contrasting methods of modeling the complex permittivity of a material in which the number of dielectric mechanisms is unknown. Using the PMF, we imposed a distribution on the resonance wavenumber and considered two approximation schemes for estimating the unknown distribution. We also considered a method which uses a convolution of Lorentz and Gaussian functions, the modified Efimov approach. We considered both simulated and experimental data sets. Within the context of the PMF, it is clear that considering the model fits alone are not sufficient to determine which approximation scheme to use, since both consistently give good model fits even if the estimated distributions vary. It is not surprising that the Dirac methods are better suited to estimate a discontinuous distribution and that the spline method are better suited to handle estimating a continuous distribution. In practice of course, there in general is no prior knowledge as to the form of the unknown distribution (continuous or discontinuous). Fortunately, we have illustrated in the examples presented in this work that the spline approximation method gives reasonable estimates even for the cases where the true distribution possesses discontinuities. Thus, it is our recommendation that initially both methods should be used to do the inverse problem. Once it is established that the estimated distributions using both methods sufficiently agree, then the results obtained using the lowest number of nodes possible to achieve this agreement should be used. This should minimize any effects of over parameterization (see Section 3.1.1). Only after the distributions agree should the decision be made as to whether the distribution appears to obtain discontinuities. If discontinuities (or regions of relative rapid change) are present in the distribution, then the results using the Dirac method would be preferred, and the results from the spline approximation would be preferred for distributions which appear continuous in nature.

Using the modified Efimov method, we were also able to obtain very good model fits to the data for both the simulated data and the inorganic glass data. It was seen that using this approach, regions of rapid increase in the distribution will correspond to oscillators with a narrow broadening. However, one pitfall to this approach is the difficulty in ascertaining the relative "importance" of each oscillator, for which the only indication is the estimated intensity. One advantage that the PMF approach has over the modified Efimov approach is that the estimated distribution can easily be interpreted. Furthermore, there is a strong theoretical foundation for the PMF approximation schemes to converge as $N \rightarrow \infty$ (with the assumption that the density function is absolutely continuous in the case of using splines), however, there is no known sense of convergence as $J \rightarrow \infty$ in the Efimov approach.

In future work, we plan to consider experimental data sets obtained from CMCs which have undergone various levels of heat treatment and attempt to use the methods described here to ascertain levels of degradation. Additionally, we would like to understand the mathematical and statistical model discrepancy and use this in an effort to quantify the uncertainty in the probability distribution estimators. 


\section{Acknowledgements}

This research was supported in part by the Air Force Office of Scientific Research under grant number AFOSR FA9550-12-1-0188, and in part by the US Department of Education Graduate Assistance in Areas of National Need (GAANN) under grant number P200A120047. The authors are gratefully to Dr. Amanda Criner and her colleagues at Wright-Patterson Air Force Base for their encouragement and insightful comments regarding this work.

\section{References}

[1] P. Baldus, M. Jansen and D. Sporn, Ceramic Fibers for Matrix Composites in HighTemperature Engine Applications, Science, 285 (1999), 699-703.

[2] H.T. Banks, A Functional Analysis Framework for Modeling, Estimation and Control in Science and Engineering, Chapman and Hall/CRC Press, Boca Raton, FL, 2012.

[3] H.T. Banks and K.L. Bihari, Modeling and estimating uncertainty in parameter estimation, Inverse Problems, 17 (2001), 95-111.

[4] H. T. Banks, J. Catenacci, and S. Hu, Estimation of distributed parameters in permittivity models of composite dielectric materials using reflectance, CRSC-TR14-08, N. C. State University, Raleigh, NC, June, 2014; J. Inverse and Ill-posed Problems, to appear.

[5] H.T. Banks, J. Catenacci and S. Hu, Asymptotic properties of probability measure estimators in a nonparametric model, SIAM/ASA Journal on Uncertainty Quantification, to appear.

[6] H.T. Banks and N.L. Gibson, Electromagnetic inverse problems involving distributions of dielectric mechanisms and parameters, Quarterly of Applied Mathematics, 64 (2006), 749-795.

[7] H.T. Banks, S. Hu, and W.C. Thompson, Modeling and Inverse Problems in the Presence of Uncertainty, Chapman \& Hall/CRC Press, Boca Raton, FL, 2014.

[8] H.T. Banks and G.A. Pinter, A probabilistic multiscale approach to hysteresis in shear wave propagation in biotissue, SIAM J. Multiscale Modeling and Simulation, 3 (2005), 395412.

[9] J.G. Blaschak and J. Fanzen, Precursor propagation in dispersive media from shortrise-time pulses at oblique incidence, Journal of the Optical Society of America A, 12 (1995), 1501-1512.

[10] A.T. Cooney, R.Y. Flattum-Riemers, and B.J. Scott, Characterization of material degradation in ceramic matrix composites using infrared reflectance spectroscopy, AIP Conference Proceedings, San Diego, California, July 18-23, 2010.

[11] A.M. Efimov, Optical Contants of Inorganic Glasses, CRC press, Boca Raton, Florida, 1995. 
[12] A.M. Efimov, Quantitative IR spectroscopy: Applications to studying glass structure and properties, Journal of Non-Crystalline Solids, 203 (1996), 1-11.

[13] A.M. Efimov, Vibrational spectra, related properties, and structure of inorganic glasses, Journal of Non-Crystalline Solids, 253 (1999), 95-118.

[14] A.M. Efimov and E. G. Makarova, Dispersion equation for the complex dielectric constant of vitreous solids and dispersion analysis of their reflection spectra, Fiz. Khim. Stekla (Journal of Applied Spectroscopy), 11, (1985), 385.

[15] L. Giacomazzi and A. Pasquarello, Vibrational spectra of vitreous $\mathrm{SiO}_{2}$ and vitreous $\mathrm{GeO}_{2}$ from first principles, Journal of Physics: Condensed Matter, 19 (2007), 112-121.

[16] S.R. Pina, L.C. Pardini, I.V. Yoshida, Carbon fiber/ceramic matrix composites: processing, oxidation and mechanical properties, Journal of Material Science, 42 (2007) 4245-4253.

[17] Yu.V. Prohorov, Convergence of random processes and limit theorems in probability theory, Theor. Prob. Appl., 1 (1956), 157-214.

[18] G. Qi, C. Zhang, H. Hu, F. Cao, S. Wang, Y. Jiang, B. Li, Crystallization behavior of three-dimensional silica fiber reinforced silicon nitride composite, Journal of Crystal Growth, 284 (2005), 293-296.

[19] H. Ohnabe, S. Masaki, M. Onozuka, K. Miyahara, T. Sasa, Potential application of ceramic matrix composites to aero-engine components, Composites Part A: Applied Sciences and Manufacturing, 30 (1999), 489-496.

[20] V. Raman, G. Bhatia, P.R. Sengupta, A.K. Srivastava, K.N. Sood, Synthesis of silicon carbide nanorods from mixture of polymer and sol-gel silica, Journal of Material Science, 42 (2007), 5891-5895. 